\title{
MAPS BETWEEN SURFACES
}

BY

RICHARD SKORA

\begin{abstract}
The Uniqueness Conjecture states if $\phi, \psi: M \rightarrow N$ are $d$-fold, simple, primitive, branched coverings between closed, connected surfaces, then $\phi$ and $\psi$ are equivalent. The Uniqueness Conjecture is proved in the case that $M$ and $N$ are nonorientable and $N=\mathbf{R} P^{2}$ or Klein bottle. It is also proved in the case that $M$ and $N$ are nonorientable and $d / 2<d \chi(N)-\chi(M)$. As an application it is shown that two $d$-fold, branched coverings $\phi: M_{1} \rightarrow N, \psi: M_{2} \rightarrow N$ between closed, connected surfaces are branched cobordant.
\end{abstract}

0. Introduction. This paper considers the following conjecture.

UNIQUENESS CONJECTURE. Let $\phi, \psi: M \rightarrow N$ be $d$-fold, simple, primitive, branched coverings between closed, connected surfaces. Then $\phi$ and $\psi$ are equivalent.

The U.C. in a certain form goes back to the 19th century when [A. Clebsch, 1873] extended the work of [J. Lüroth, 1871] to prove the conjecture in the case that $N=S^{2}$. The U.C. was first stated in the above generality by [I. Berstein and A. Edmonds, 1984]. In the orientable case they prove the conjecture when $N=S^{1} \times S^{1}$ or $d / 2<d \chi(N)-\chi(M)$. For a more complete history of the conjecture see [Berstein and Edmonds, 1984] and [Skora, 1984].

The contents of this paper are as follows. $\$ 1$ contains definitions and background theorems. $\$ 2$ contains an example showing the necessity of the simple hypothesis in the Uniqueness Conjecture. In $\$ 3$ the Uniqueness Conjecture is proved in the case that $M$ and $N$ are nonorientable and $N=\mathbf{R} P^{1}$ or Klein bottle. It is also proved when $M$ and $N$ are nonorientable and $d / 2<d \chi(N)-\chi(M)$. In $\S 4$ it is proved that two $d$-fold, branched coverings $\phi: M_{1} \rightarrow N, \psi: M_{2} \rightarrow N$ between closed, connected surfaces are branched cobordant.

The results in this paper are from the author's dissertation [Skora, 1984]. I thank my advisor Cameron Gordon for his guidance. I am also grateful to Andrew Casson and Gary Hamrick for their help.

1. Definitions and background theorems. More details to the following definitions and theorems may be found in [Berstein and Edmonds, 1984].

Definition. Maps $\phi, \psi: X \rightarrow Y$ of topological spaces are equivalent if there are homeomorphisms $f: X \rightarrow X$ and $g: Y \rightarrow Y$ such that $g \circ \phi=\psi \circ f$.

Received by the editors October 2, 1984 and, in revised form, January 14, 1985.

1980 Mathematics Subject Classification. Primary 57M12; Secondary 57N05.

Key words and phrases. Branched covering, Uniqueness Conjecture, branched cobordant.

(C)1985 American Mathematical Society $0002-9939 / 85 \$ 1.00+\$ .25$ per page 
Definition. A simplicial map $\phi: X \rightarrow Y$ of $n$-manifolds is a branched covering if $\phi \mid X \backslash X^{n-2}: X \backslash X^{n-2} \rightarrow Y \backslash Y^{n-2}$ is a covering, where $X^{n-2}, Y^{n-2}$ are the $(n-$ 2)-skeletons. Let $X_{0}, Y_{0}$ be the largest open subsets of $X$ and $Y$ such that $\phi \mid X_{0}$ : $X_{0} \rightarrow Y_{0}$ is a covering. Then $\phi_{0}=\phi \mid X_{0}$ is the associated covering, and $\phi$ is called $d$-fold if $\phi_{0}$ is $d$-fold. The singular set $\Sigma_{\phi}=X \backslash X_{0}$ and the branch set $B_{\phi}=Y \backslash Y_{0}$.

In the case that $\phi: M \rightarrow N$ is a branched covering of surfaces, $\phi$ looks like a wrap map $z \rightarrow z^{n}$ of the complex plane, where $2 \leqslant n$, in a suitably chosen neighborhood of a singular point. The order of $x \in \Sigma_{\phi}$ is $n-1$. The classical Riemann-Hurwitz formula is $\sum_{x \in \Sigma_{\phi}} \operatorname{order}(x)=d \chi(N)-\chi(M)$, where $M$ and $N$ are compact and $\phi$ is $d$-fold.

Notice that $d \chi(N)-\chi(M)$ measures the difference between $\phi$ and a covering of the same number of sheets. When $M$ and $N$ are closed, it is a consequence of the relations of a presentation for $\pi_{1}(N)$ that $d \chi(N)-\chi(M)$ is even.

Let $\phi: X \rightarrow Y$ be a covering of topological spaces and $* \in Y, \Omega=\phi^{-1}(*)$. If $\alpha$ is a loop in $Y$ based at $*$, then $[\alpha] \in \pi_{1}(Y, *)$ and a lift of $\alpha$ will be a path $\tilde{\alpha}$ in $X$ with both $\tilde{\alpha}(0)$ and $\tilde{\alpha}(1)$ in $\Omega$. Then $[\alpha]$ defines a right action on $\Omega$ given by $\tilde{\alpha}(0) \rightarrow \tilde{\alpha}(1)$, where $\tilde{\alpha}$ ranges over all lifts of $\alpha$. Let $S_{\Omega}$ be the symmetric group of $\Omega$ also acting on the right. One has a well-defined representation $\pi_{1}(Y, *) \rightarrow S_{\Omega}$. If $\phi$ is $d$-fold and one labels the elements of $\Omega$ by the letters $\{1,2, \ldots, d\}$, then the representation $\pi_{1}(Y, *)$ $\rightarrow S_{d}$ is unique up to inner automorphism of $S_{d}$. Either representation is called the associated representation and is denoted $\rho_{\phi}$. If $\phi: X \rightarrow Y$ is a branched covering, then $\rho_{\phi_{0}}$ is the associated representation and is denoted $\rho_{\phi}$. The following theorem due to [A. Hurwitz, 1891] is easy by modern standards.

1.1. Classification Theorem. Let $\phi_{i}:\left(X, x_{i}\right) \rightarrow\left(Y, y_{i}\right), i=1,2$, be $d$-fold branched coverings. Then $\phi_{1}$ and $\phi_{2}$ are equivalent iff there are a homeomorphism $g$ : $\left(Y, B_{\phi_{1}}, y_{1}\right) \rightarrow\left(Y, B_{\phi_{2}}, y_{2}\right)$ and inner automorphism $a: S_{d} \rightarrow S_{d}$ such that $a \circ \rho_{\phi_{1}}=$ $\rho_{\phi_{2}} \circ g_{*}$.

Definition. A $d$-fold branched covering $\phi: X \rightarrow Y$ is simple if $\left|\phi^{-1}(x)\right|=d-1$ for all $x \in B_{\phi}$.

The motivation for studying simple branched coverings is the following theorem from [Berstein and Edmonds, 1979]. Let $C(M, N)$ be the function space of branched coverings with the compact open topology.

1.2. TheOREM. Let $M$ and $N$ be compact, connected surfaces. Then the set of simple, branched coverings of $N$ by $M$ is an open, dense subset of $C(M, N)$.

Definition. A branched covering $\phi: X \rightarrow Y$ of connected manifolds is primitive if $\phi_{*}$ is surjective on $\pi_{1}$.

The following is from [Berstein and Edmonds, 1984].

1.3. TheOREM. Let $\phi: X \rightarrow Y$ be a simple, branched covering with $B_{\phi} \neq \varnothing$. Then $\phi$ is primitive if and only if $\rho_{\phi}$ is surjective. 
2. Example. In this section an example is mentioned which demonstrates the necessity of the simple hypothesis in the Uniqueness Conjecture.

Let $M$ be the genus-4, closed, orientable surface. Then up to equivalence there are exactly two regular, 3-fold, primitive branched coverings $\phi, \psi: M \rightarrow S^{2}$. Necessarily each branch point is of order 2 and $\left|B_{\phi}\right|=\left|B_{\psi}\right|=6$. They may be constructed by taking representations into $\mathbf{Z} / 3 \mathbf{Z}$ and distinguished by the action of the deck translations near the branch points.

3. On the Uniqueness Conjecture. In this section the Uniqueness Conjecture is proved in the case that $M$ and $N$ are nonorientable and $N=\mathbf{R} P^{2}$ or Klein bottle. And it is proved in the case that $M$ and $N$ are nonorientable and $d / 2<d \chi(N)-$ $\chi(M)$. The definitions and methods are generalized from [Berstein and Edmonds, 1984].

Given a branched covering $\phi: M \rightarrow N$ of surfaces, it is completely determined by its associated representation $\rho_{\phi}: \pi_{1}\left(N \backslash B_{\phi}, *\right) \rightarrow S_{\Omega}$. So it will be convenient to encode the information of the representation.

Definition. If $\phi: M \rightarrow N$ is a branched covering, where $N$ is a compact surface, a Hurwitz graph for $\phi$ is a finite, oriented graph $\Gamma$ with one vertex $*$ and an embedding $(\Gamma, *) \rightarrow\left(N \backslash B_{\phi}, *\right)$ such that $\pi_{1}(\Gamma) \rightarrow \pi_{1}\left(N \backslash B_{\phi}\right)$ is surjective. A Hurwitz system for $\phi$ is a pair $(\mathscr{H}, \Gamma)$, where $\Gamma$ is a Hurwitz graph and $\mathscr{H}$ is a sequence of permutations in $S_{\Omega}$ corresponding to the representations of the oriented edges of $\Gamma$.

Notice that $(\mathscr{H}, \Gamma)$ is not unique. Figure 3.1 shows a Hurwitz graph in Klein bottle \# R $P^{2} \# \cdots \# \mathbf{R} P^{2}$. One says two Hurwitz systems $\left(\mathscr{H}_{1}, \Gamma_{1}\right)$ and $\left(\mathscr{H}_{2}, \Gamma_{2}\right)$ for $\phi_{1}: M_{1} \rightarrow N_{1}$ and $\phi_{2}: M_{2} \rightarrow N_{2}$ are the same if there is a homeomorphism $\left(N_{1}, B_{\phi_{1}}\right) \rightarrow\left(N_{2}, B_{\phi_{2}}\right)$, taking $\Gamma_{1}$ to $\Gamma_{2}$ respecting the orientation and respecting $\mathscr{H}_{1}$

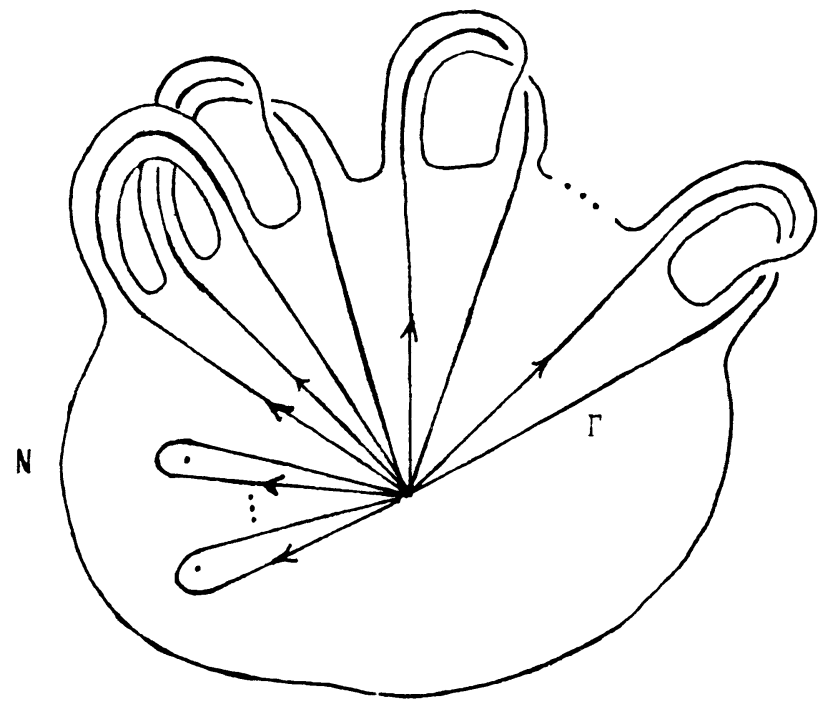

Klein bottle \# R $P^{2} \# \cdots \# \mathbf{R} P^{2}$

FIGURE 3.1 
and $\mathscr{H}_{2}$. So one has the following easy reformulation of Hurwitz's Classification Theorem.

3.1. THEOREM. Two branched coverings of compact surfaces are equivalent if and only if they have the same Hurwitz systems.

Since the only concern is for simple, branched coverings $\phi: M \rightarrow N$, where $N$ is closed and nonorientable, the Hurwitz graph $\Gamma$ never need be more general than the one pictured in Figure 3.1, so suppose $\left(N, \Gamma, B_{\phi}\right)$ is equivalent as a triple to that in Figure 3.1. Then the equivalence class of the branched covering is actually determined by $\mathscr{H}$ alone, where $\mathscr{H}$ will be of the form $\left(\sigma_{1}, \ldots, \sigma_{n} ; \alpha, \delta_{0}, \delta_{1}, \ldots, \delta_{x}\right)$. The $\sigma_{i}$ 's correspond to branch points, the pair $\alpha, \delta_{0}$ is a Klein bottle and the $\delta_{j}$ 's, $1 \leqslant j$, are $\mathbf{R} P^{2}$ 's. In particular $\left(\sigma_{1}, \ldots, \sigma_{n} ; \delta\right),\left(\sigma_{1}, \ldots, \sigma_{n} ; \alpha, \delta\right)$ and $\left(\sigma_{1}, \ldots, \sigma_{n}\right.$; $\left.\alpha, \delta_{0}, \delta_{1}, \ldots, \delta_{x}\right)$ denote Hurwitz systems for simple branched coverings $\phi: M \rightarrow N$, where $N$ is $\mathbf{R} P^{2}$, Klein bottle and a nonorientable closed surface with Euler characteristic $-x$, respectively. Notice that $\sigma_{i}$ is always a transposition; the systems satisfy the relations $\sigma_{1} \cdots \sigma_{n} \delta^{2}=1, \sigma_{1} \cdots \sigma_{n} \alpha \delta^{-1} \alpha \delta=1$ and $\sigma_{1} \cdots \sigma_{n} \alpha \delta_{0}^{-1} \alpha \delta_{0} \delta_{1}^{2} \cdots$ $\delta_{x}^{2}=1$; and $n$ is even.

The strategy in proving the Uniqueness Conjecture is to define a canonical sequence $\mathscr{H}^{*}$ for a branched covering. Then one shows that the branched covering has a sequence $\mathscr{H}$ (corresponding to some Hurwitz graph $\Gamma$ ) such that $\mathscr{H}$ may be deformed to $\mathscr{H}^{*}$ by the following moves. These moves on $\mathscr{H}$ are induced by reembeddings of $\Gamma$. The moves to $\mathscr{H}$ are described below without reference to $\Gamma$ and only the affected part of $\mathscr{H}$ is mentioned. See [Berstein and Edmonds, 1984] for more details.

Recall that if $a, b$ are group elements, then $a^{b}=b a b^{-1}$. If $g \in S_{\Omega}$, then the set of elements of $\Omega$ not fixed by $g$ is the support of $g$ and is denoted $|g|$.

Move 1. Here $\sigma$ and $\tau$ are transpositions corresponding to branch points: $(\sigma, \tau) \rightarrow$ $\left(\tau^{\sigma}, \sigma\right),(\sigma, \tau) \rightarrow\left(\tau, \sigma^{\tau}\right)$.

Move 2. $(\sigma ; \alpha, \delta) \rightarrow\left(\sigma^{\sigma \alpha \delta^{-1}} ; \sigma \alpha, \delta\right)$.

Move 3. $(\sigma ; \alpha, \delta) \rightarrow\left(\sigma^{\sigma \alpha} ; \alpha^{\sigma}, \sigma \delta\right)$.

When Move 3 is used to change $\sigma$, the effect on $\alpha$ (and $\delta$ ) is undesirable. It is important to understand exactly what this side effect is. First notice that $\alpha^{\alpha}=\alpha^{\sigma \alpha}$ and $\sigma^{\sigma \alpha} \alpha^{\sigma}=\sigma \alpha$, that is to say the product $\sigma \alpha$ is preserved by Move 3. So one may prefer to write it as

$$
(\sigma ; \alpha, \delta) \rightarrow\left(\sigma^{\sigma \alpha} ; \alpha^{\sigma \alpha}, \sigma \alpha\right) .
$$

In typical applications, $|\sigma| \subset$ a single orbit of $\alpha$, so $\sigma \alpha$ has one more orbit than $\alpha$. The following example shows that this move has predictable effects on $\alpha$. Start with

$$
((15) ;(12345678), \delta) \text {. }
$$

The bracket reminds one that $(15)(12345678)=(1678)(2345)$. Four applications of Move 3 give the following Hurwitz systems:

$$
\begin{aligned}
& \left((84) ;(85234167), \delta^{\prime}\right), \\
& \left((73) ;(74523816), \delta^{\prime \prime}\right), \\
& \left((62) ;(63452781), \delta^{\prime \prime \prime}\right), \\
& \left((15) ;(12345678), \delta^{\prime \prime \prime \prime}\right) .
\end{aligned}
$$


Move 4. $(\sigma ; \delta) \rightarrow\left(\sigma^{\sigma \delta} ; \sigma \delta\right)$.

Move 5. $(\alpha, \delta) \rightarrow\left(\alpha, \alpha^{k} \delta\right)$.

Move 6. This move is intermediate and used only to conjugate Moves 3 or 4 . Move 6 includes the following and their inverses:

$$
\begin{aligned}
& \left(\alpha, \delta_{0}, \delta_{1}\right) \rightarrow\left(\delta_{1}^{\alpha \delta_{0}^{-1} \alpha \delta_{0}}, \alpha, \delta_{0}\right), \\
& \left(\alpha, \delta_{0}, \delta_{1}\right) \rightarrow\left(\delta_{1}, \alpha^{\delta_{1}^{-2}}, \delta_{0}^{\delta_{1}^{-2}}\right), \\
& \left(\delta_{i}, \delta_{i+1}\right) \rightarrow\left(\delta_{i+1}^{\delta_{i}^{2}}, \delta_{i}\right) .
\end{aligned}
$$

Certain sequences of the above moves will be used frequently enough to justify

3.2. Doubles Lemma.

$$
\begin{aligned}
& (\sigma, \sigma, \tau) \rightarrow\left(\sigma^{\tau}, \sigma^{\tau}, \tau\right), \\
& (\tau, \sigma, \sigma) \rightarrow\left(\tau, \sigma^{\tau}, \sigma^{\tau}\right), \\
& (\sigma, \sigma ; \alpha, \delta) \rightarrow\left(\sigma^{\alpha \delta^{-1}}, \sigma^{\alpha \delta^{-1}} ; \alpha, \delta\right), \\
& (\sigma, \sigma ; \alpha, \delta) \rightarrow\left(\sigma^{\alpha}, \sigma^{\alpha} ; \alpha, \delta\right), \\
& (\sigma, \sigma ; \alpha, \delta) \rightarrow\left(\sigma^{\delta^{-1}}, \sigma^{\delta^{-1}} ; \alpha, \delta\right), \\
& (\sigma, \sigma ; \delta) \rightarrow\left(\sigma^{\delta}, \sigma^{\delta} ; \delta\right) .
\end{aligned}
$$

Proof. The first statement uses only Move $1:(\sigma, \sigma, \tau) \rightarrow\left(\sigma, \tau^{\sigma}, \sigma\right) \rightarrow(\tau, \sigma, \sigma) \rightarrow$ $\left(\sigma^{\tau}, \tau, \sigma\right) \rightarrow\left(\sigma^{\tau}, \sigma^{\tau}, \tau\right)$. The others are proved similarly.

It may be instructive to see directly by picture how these doubles moves correspond to reembeddings of the Hurwitz graph. That it works depends strongly on that $\sigma$ and $\tau$ are transpositions.

Now follows the proof of the Uniqueness Conjecture when $N=\mathbf{R} P^{2}$ or Klein bottle.

3.3. Lemma. Let $\phi: M \rightarrow \mathbf{R} P^{2}$ be a $d$-fold, simple, primitive branched covering. Then $\phi$ has a Hurwitz system $,(12),(23),(34), \ldots,(d-1 d)$;

$$
(\cdots d-2(d-3) / 2 d-1(d-1) / 2 d))
$$

when $d$ is odd and a Hurwitz system

$$
\begin{array}{r}
((12), \ldots,(12),(23),(34), \ldots,(d / 2 d / 2+1),(d / 2 d / 2+1), \ldots,(d-1 d) ; \\
(\cdots(d-2) / 2 d-1 d / 2 d))
\end{array}
$$

when $d$ is even.

Proof. Let $\left(\sigma_{1}, \ldots, \sigma_{n} ; \delta\right)$ be any Hurwitz system for $\phi$. If $\delta$ is not a $d$-cycle, then transitivity of the associated representation implies that for some $i, 1 \leqslant i \leqslant n, \sigma_{i} \delta$ has fewer orbits than $\delta$. By Move 1 suppose $i=n$ and by Move 4 reduce the number of orbits of $\delta$. Continuing in this way one arrives at a Hurwitz system with $\delta$ a $d$-cycle. Continue to denote the new system by $\left(\sigma_{1}, \ldots, \sigma_{n} ; \delta\right)$. 
Using Move 1 , a Hurwitz system $\left(\mathscr{S}_{1}, \mathscr{S}_{2} ; \delta\right)$ is produced, where $\mathscr{S}_{1}$ and $\mathscr{S}_{2}$ are sequences of transpositions, and $\mathscr{S}_{2}$ is exactly those transpositions in the system which permute the letter $d$. If $\mathscr{S}_{2}$ contains two different transpositions, then apply Move 1 to change at least one of the transpositions of $\mathscr{S}_{2}$ into one which fixes $d$, and then reduce the length of $\mathscr{S}_{2}$. So suppose $\mathscr{S}_{2}$ has the additional property that it is a sequence of identical transpositions. If necessary, conjugate the representation to obtain a Hurwitz system $\left(\mathscr{S}_{1},(d-1 d), \ldots,(d-1 d) ; \delta\right)$.

Now induction gives a Hurwitz system $\left(n_{1}(12), n_{2}(23), \ldots, n_{d-1}(d-1 d) ; \delta\right)$, where $n_{i}(i i+1)$ is a sequence of $(i i+1)$ 's of length $n_{i}$. From here there are two cases.

If $d$ is odd, then $\delta^{2}$ is a $d$-cycle. The relation $\sigma_{1} \cdots \sigma_{n} \delta^{2}=1$ implies that each $n_{i}$ is odd. Suppose $2<n_{i}$ for some $i>1$. Then there is a subsequence $((i-1 i),(i i+1)$, $(i i+1),(i i+1))$. Apply the Doubles Lemma to get $((i-1 i),(i-1 i+1)$, $(i-1 i+1),(i i+1))$ and apply it again to get $((i-1 i),(i-1 i),(i-1 i)$, $(i i+1))$. This has the effect of shuffling excess transpositions to the left. Continuing in this way produces a Hurwitz system $((12), \ldots,(12),(23), \ldots,(d-1 d) ; \delta)$. The relation $\sigma_{1} \cdots \sigma_{n} \delta^{2}=1$ implies that $\delta=(\cdots d-2(d-3) / 2 d-1(d-1) / 2 d)$ as claimed.

If $d$ is even, then $\delta^{2}$ is the product of two disjoint $d / 2$-cycles. This time the relation $\sigma_{1} \cdots \sigma_{n} \delta^{2}=1$ implies that $n_{i}$ is odd iff $i \neq d / 2$. Shuffling excess transpositions to the left as above produces the Hurwitz system $((12), \ldots,(12)$, (2 3), $\left.(34), \ldots, n_{d / 2}(d / 2 d / 2+1), \ldots,(d-1 d) ; \delta\right)$, where $n_{d / 2}=0$ or 2 . Since $\phi$ is primitive, Theorem 1.3 says the representation is surjective. So $n_{d / 2}=2$. But the relation $\sigma_{1} \cdots \sigma_{n} \delta^{2}=1$ does not determine $\delta$ uniquely.

Suppose $\delta=(\cdots d-1 k d)$, where $1 \leqslant k \leqslant d / 2$. Now apply Move 1 and the Doubles Lemma only to those transpositions to the left of and including the pair $(d / 2 d / 2+1),(d / 2 d / 2+1)$ to find a Hurwitz system $\left(\mathscr{S}_{1},(k d / 2+1),(k\right.$ $d / 2+1), \ldots,(d-1 d) ;(\cdots d-1 k d))$. Change the system to $\left(\mathscr{S}_{1}^{\prime},(d / 2 d / 2+1)\right.$, $(d / 2 d / 2+1), \ldots,(d-1 d) ;(\cdots d-1 d / 2 d))$ by conjugating $k$ to $d / 2$. Work on $\mathscr{S}_{1}$ as above to produce the desired Hurwitz system.

3.4. TheOREM. Let $\phi, \psi: M \rightarrow \mathbf{R} P^{2}$ be $d$-fold, simple, primitive branched coverings. Then $\phi$ and $\psi$ are equivalent.

Proof. By Lemma 3.3, $\phi$ and $\psi$ have the same Hurwitz systems. By Theorem 3.1, $\phi$ and $\psi$ are equivalent.

3.5. Lemma. Let $\phi: M \rightarrow$ Klein bottle be a $d$-fold, simple, primitive branched covering. Then $\phi$ has a Hurwitz system $((12), \ldots,(12) ;(12 \cdots d),(1 d)(2 d-1)$ $\cdots)$.

Proof. Choose a Hurwitz system $\left(\sigma_{1}, \ldots, \sigma_{n} ; \alpha, \delta\right)$ for $\phi$ such that the number of orbits of $\alpha$ is minimized over all such systems. It is claimed that $\alpha$ is a $d$-cycle. By the choice of $\alpha$ and the existence of Moves 1 and 2, the support of each $\sigma_{i}$ is contained in a single orbit of $\alpha$. If $\left\{P_{j}\right\}_{j}$ is a partitioning of the letters $1,2, \ldots, d$ into the orbits of 
$\alpha$, then $P_{j} \sigma_{i}=P_{j}$ for all $i, j$. That is, the $\sigma_{i}$ 's respect the partitioning, and the orbits of $\sigma_{1} \cdots \sigma_{n}$ are subsets of the orbits of $\alpha$. The relation $\sigma_{1} \cdots \sigma_{n} \alpha=\delta^{-1} \alpha^{-1} \delta$ says that $\sigma_{1} \cdots \sigma_{n} \alpha$ is conjugate to $\alpha^{-1}$ which is conjugate to $\alpha$. So the orbits of $\sigma_{1} \cdots \sigma_{n} \alpha$ are exactly the orbits of $\alpha$. And the orbits of $\delta^{-1} \alpha^{-1} \delta$ are exactly the orbits of $\alpha^{-1}$. So $\delta$ also respects the partitioning. By primitivity and Theorem $1.3, \alpha$ is a $d$-cycle.

Next $i<n$ is found such that $\sigma_{i} \sigma_{n} \alpha$ is conjugate to $\alpha$. Since $\alpha$ is a $d$-cycle, $\sigma_{n} \alpha$ has one more orbit than $\alpha$. In general $\sigma_{i} \cdots \sigma_{n} \alpha$ has one more or one less orbit than $\sigma_{i+1} \cdots \sigma_{n} \alpha$. Since $\sigma_{1} \cdots \sigma_{n} \alpha$ is also a $d$-cycle, there is some $i<n$ such that $\sigma_{i} \sigma_{n} \alpha$ is conjugate to $\alpha$.

Use Move 1 to get a new Hurwitz system, still denoted $\left(\sigma_{1}, \ldots, \sigma_{n} ; \alpha, \delta\right)$, such that $\sigma_{n-1} \sigma_{n} \alpha$ is conjugate to $\alpha$. By Lemma 3.6 below one may suppose that $\sigma_{n-1}=\sigma_{n}$ and $\alpha$ is still a $d$-cycle. By shuffling $\sigma_{n-1}$ and $\sigma_{n}$ out of the way, one may repeatedly apply the above reasoning to find a Hurwitz system with $\sigma_{2 k-1}=\sigma_{2 k}$ and $\alpha$ still a $d$-cycle.

Now apply Lemma 3.7 below to find a Hurwitz system $((1 \mathrm{~m}), \ldots,(1 \mathrm{~m}) ;(12 \ldots$ $d), \delta$ ). The relation $\alpha=\delta^{-1} \alpha^{-1} \delta$ implies $\langle\alpha, \delta\rangle$ is the dihedral group of degree $d$. By primitivity and Lemma 3.8, $d$ and $m-1$ are relatively prime.

If $m \neq 2$, alter the system as follows. Concentrating on $\sigma_{n-1}, \sigma_{n}$ and $\alpha$ one starts with $((1 m),(1 m) ;(12 \cdots m \cdot d))$. Since $d$ and $m-1$ are relatively prime, applications of Move 3 will produce $((1 m),(12),(1 \sqrt{3 \cdots m} 2 \cdots d))$. Move 1 gives

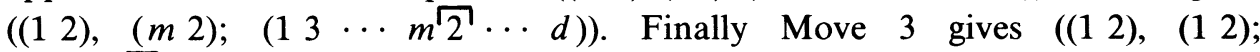
$(\cdots d 1 \sqrt{2} 3 \cdots))$. Clearly one may now find a Hurwitz system $((12), \ldots,(12)$; $(12 \cdots d), \delta)$. If necessary, apply Move 5 to fix $\delta$ and get the desired canonical form.

3.6. Lemma. Let $(\ldots, \sigma, \tau ; \alpha, \delta, \ldots)$ be a Hurwitz system for a simple branched covering $\phi$ such that $|\sigma|$ and $|\tau|$ lie in the same orbit of $\alpha$, and $\sigma \tau \alpha$ is conjugate to $\alpha$. Then $\phi$ has a Hurwitz system $\left(\ldots, \sigma^{\prime}, \sigma^{\prime} ; \alpha^{\prime}, \delta^{\prime}, \ldots\right)$ where $\alpha^{\prime}$ is conjugate to $\alpha$ and the only changes to the system are those shown.

Proof. Notice that the product $\sigma \tau \alpha$ is preserved by Moves 1 and 3. In particular Move 3 alone will give a Hurwitz system which still satisfies the hypothesis and $|\sigma| \cap|\tau| \neq \varnothing$. Also only the orbit of $\alpha$ containing $|\sigma|$ and $|\tau|$ is affected by the moves. Without loss of generality suppose the given Hurwitz system is $((1 i),(1 j)$; $(12 \cdots d), \delta)$. That $\sigma \tau \alpha$ is conjugate to $\alpha$ is equivalent to $i \leqslant j$. If $i \neq j$, then proceed as follows.

If $j=d$, then $d-i$ applications of Move 3 gives a Hurwitz system ((1i); (1i); $\left.\alpha^{\prime}, \delta^{\prime}\right)$ and the argument is complete.

Now induct on $d-j$. Suppose $i<j<d$ and the Hurwitz system is $((1 i),(1 j)$; $(1 \cdots i \cdots j \cdots d), \delta)$. Apply Move $3, j-i$ times. If one is lucky enough that $d-j+1$ divides $j-i$, one immediately finds the Hurwitz system $((1 i),(1 i)$; $\left.(1 \cdots i \cdots d), \delta^{\prime}\right)$. If $d-j+1$ does not divide $j-i$, one finds $((1 i),(j+r i)$; $\left.(j+r \cdots j \cdots i \cdots 1 \cdots), \delta^{\prime}\right)$. Apply Move 1 to get $((j+r i),(j+r 1) ;(j+$ $\left.r \cdots j \cdots i \cdots 1 \cdots), \delta^{\prime \prime}\right)$. Conjugating the representation gives $((1 j),(1 j+s)$; $\left.(12 \cdots d), \delta^{\prime \prime \prime}\right)$. It is clear that $d-(j+s)<d-j$. 
3.7. LEMMA. If $\left(\sigma_{1}, \ldots, \sigma_{n} ; \alpha, \delta\right)$ is a Hurwitz system for a $d$-fold, simple branched covering $\phi, \alpha$ is a d-cycle and $\sigma_{2 k-1}=\sigma_{2 k}$ for $1 \leqslant k \leqslant n / 2$, then $\phi$ has a Hurwitz $\operatorname{system}(\sigma, \sigma, \ldots, \sigma ; \alpha, \delta)$.

Proof. Suppose without loss of generality the given Hurwitz system is $\left(\left(1 i_{1}\right)\right.$, $\left.\left(1 i_{1}\right), \ldots,\left(1 i_{k}\right),\left(1 i_{k}\right) ;(12 \cdots d), \delta\right)$. Suppose $i_{l}<i_{m}$ for some $l$ and $m$. Using Move 1 and the Doubles Lemma, one may change a pair of $\left(1 i_{m}\right)$ 's to $\left(i_{l} i_{m}\right)$ 's. Using $\alpha$ and the Doubles Lemma, one changes the pair of $\left(i_{l} i_{m}\right)$ 's to $\left(1 i_{m}-i_{l}\right)$. Inducting on $\sum_{l} i_{l}$ finishes the proof.

The following is elementary.

3.8. Lemma. Let $D<S_{d}$ be the dihedral group of degree d containing (12 $\cdots d$ ). If $(1 \mathrm{~m})$ and $D$ generate $S_{d}$, then $m-1$ and $d$ are relatively prime.

3.9. TheOREM. Let $\phi, \psi: M \rightarrow$ Klein bottle be $d$-fold, simple, primitive branched coverings. Then $\phi$ and $\psi$ are equivalent.

Next is the classification of simple, primitive, branched coverings of a closed, nonorientable surface with negative Euler characteristic satisfying the following

Definition. Let $\phi: M \rightarrow N$ be a $d$-fold branched covering between closed surfaces. Say that $\phi$ is metastable if $d / 2<d \chi(N)-\chi(M)$.

Recall that $\sum_{X \in \Sigma_{\phi}} \operatorname{order}(x)=d \chi(N)-\chi(M)$. So in the case of a simple branched covering, $d \chi(N)-\chi(M)$ is the number of branch points. The following proof works because there are enough branch points.

3.10. Lemma. Let $\phi: M \rightarrow N$ be a d-fold, metastable, simple, primitive branched covering where $N$ is a closed, nonorientable surface with negative Euler characteristic. Then $\phi$ has a Hurwitz system $\left(\sigma_{1}, \ldots, \sigma_{n} ; \alpha, \delta_{0}, \delta_{1}, \ldots, \delta_{x}\right)$ such that the number of orbits of $\alpha$ is minimized over all systems, and $\sigma_{n-1}=\sigma_{n}$.

Proof. Start with a Hurwitz system $\mathscr{H}=\left(\sigma_{1}, \ldots, \sigma_{n} ; \alpha, \delta\right)$ with the number of orbits of $\alpha$ minimized. Considering Moves 1 and 2, $\left|\sigma_{i}\right|$ is contained in a single orbit of $\alpha$ for each $i$. By metastability two $\sigma_{i}$ 's have support in the same orbit of $\alpha$. Without loss of generality suppose they are $\sigma_{n-1}$ and $\sigma_{n}$. If $\sigma_{n-1} \sigma_{n} \alpha$ is conjugate to $\alpha$, then the argument is completed by Lemma 3.6. So suppose $\sigma_{n-1} \sigma_{n} \alpha$ is not conjugate to $\alpha$; the goal is to find a Hurwitz system to which Lemma 3.6 applies.

Let $\mathscr{H}=\left(\sigma_{n-1}, \sigma_{n} ; \alpha, \delta\right)$ be the relevant part of the Hurwitz system. Changing $\sigma_{n-1}$ and $\sigma_{n}$ by Move 3 also inconveniently changes $\alpha$ (in fact a converse to Lemma 3.6 is true). Given $\mathscr{H}$ define another Hurwitz system for $\phi$ by $\mathscr{H}^{*}=\left(\sigma_{n-1}, \sigma_{n} ; \alpha^{*}, \delta^{*}\right)$, where $\alpha^{*}=\alpha^{\delta^{-1}}$ and $\delta^{*}=\left(\delta^{-1}\right)^{\delta^{-1} \alpha^{-1}}$. Moves 1 and 3 applied to $\mathscr{H}$ do not change $\alpha^{*}$ of $\mathscr{H}^{*}$. The object is to use Moves 1 and 3 on $\mathscr{H}$ to make Lemma 3.6 apply to $\mathscr{H}^{*}$.

Without loss of generality suppose $\left|\sigma_{n-1}\right| \cap\left|\sigma_{n}\right| \neq \varnothing$ and the relevant part of the Hurwitz system for $\phi$ is $((1 r),(1 s) ; \alpha, \delta)$. Since $\alpha$ is maximal, $|(1 r)|$ and $|(1 s)|$ lie in the same orbit of $\alpha^{*}$. If $(1 r)(1 s) \alpha^{*}$ is conjugate to $\alpha^{*}$, then one is done. If not, then $\mathscr{H}^{*}$ must be $\left((1 r),(1 s) ;(1 \cdots s \cdots r \cdots), \delta^{*}\right)$.

Apply Move 1 to $\mathscr{H}$ to get a new $\mathscr{H}=((1 s),(s r) ;(1 \cdots s \cdots r \cdots), \delta)$ and Move 3 to get $\mathscr{H}=((1 s),(1 t) ;(\cdots), \delta)$. If $(1 s)(1 t) \alpha^{*}$ is conjugate to $\alpha^{*}$, then one 
is done. If not, then $\mathscr{H}^{*}$ must be $\left((1 s),(1 t) ;(1 \cdots t \cdots s \cdots r \cdots), \delta^{*}\right)$. By the finiteness of the situation, eventually the right $\sigma_{n-1}$ and $\sigma_{n}$ pair is found.

3.11. Lemma. Let $\phi: M \rightarrow N$ be a d-fold, metastable, simple, primitive branched covering where $N$ is a closed, nonorientable surface of negative Euler characteristic. Then $\phi$ has a Hurwitz system $((12), \ldots,(12) ;(12 \cdots d),(1 d)(2 d-1) \cdots,()$, ()$, \ldots)$.

Proof. By Lemma 3.10 start with a Hurwitz system $\left(\sigma_{1}, \ldots, \sigma_{n} ; \alpha, \delta_{0}, \delta_{1}, \ldots, \delta_{x}\right)$ with the number of orbits of $\alpha$ minimized and $\sigma_{n-1}=\sigma_{n}$. Now argue that $\alpha$ is a $d$-cycle.

Let $G$ be the subgroup $\left\langle\sigma_{1}, \ldots, \sigma_{n-2}, \alpha, \delta_{0}, \ldots, \delta_{x}\right\rangle$. Let $H$ be the subgroup $\left\langle\sigma_{n}^{\gamma}\right\rangle_{\gamma \in G}$. By primitivity and Theorem $1.3,\left\langle\sigma_{n}, G\right\rangle=S_{d}$. So $H$ is a normal subgroup. Since $H$ contains a transposition, $H=S_{d}$. In particular, if $\alpha$ is not a $d$-cycle, then there is a $\gamma \in G$ such that $\left|\sigma_{n}^{\gamma}\right|$ spans two orbits of $\alpha$. By Move 2 this contradicts the maximality of $\alpha$. So $\alpha$ is a $d$-cycle.

By Lemma 3.7 and the proof of Lemma $3.10 \phi$ has a Hurwitz system $\left((1 \mathrm{~m}), \ldots,(1 \mathrm{~m}) ;(12 \cdots d), \delta_{0}, \ldots, \delta_{x}\right)$. Next it will be argued that a system with $\delta_{1}=\cdots=\delta_{x}=$ ( ) may be found. Then the proof for the Klein bottle finishes the proof.

Suppose $\delta_{l} \neq($ ) for some $1 \leqslant l \leqslant x$. It will be shown how to increase the number of orbits of $\delta_{l}$ without affecting $\alpha$ or the other $\delta_{j}$ 's. Let $G$ be as above. If $4 \leqslant d$, then $4 \leqslant n$. By Theorem $1.3, G=S_{d}$. If $d \leqslant 3$, then at least $(1 \cdots d) \in G$. In any case $\left\{(1 \mathrm{~m})^{g}\right\}_{g \in G}$ is all transpositions. By the Doubles Lemma and Move 4 one decreases the number of orbits of $\delta_{l}$. Damage done to the $\sigma_{i}$ 's may be repaired as in Lemma 3.10 and above without changing $\delta_{1}, \ldots, \delta_{x}$. Induction completes the proof.

3.12. Theorem. Let $\phi, \psi: M \rightarrow N$ be $d$-fold, metastable, simple, primitive branched coverings where $N$ is a closed, nonorientable surface of negative Euler characteristic. Then $\phi$ and $\psi$ are equivalent.

In particular the above theorems imply the Uniqueness Conjecture in the nonorientable case when $d=3$. As in the orientable case [Berstein and Edmonds, 1984] the Uniqueness Conjecture in the nonorientable case is true when $d=4,5$. This may be verified by enumerating the small number of possibilities for Hurwitz systems and using the above techniques.

Question. Is the Uniqueness Conjecture true in the range $0<d \chi(N)-\chi(M) \leqslant$ $d / 2$ ?

4. Applications. Below it is proved that if $\phi: M_{1} \rightarrow N$ and $\psi: M_{2} \rightarrow N$ are $d$-fold branched coverings between closed, connected surfaces, then $\phi$ and $\psi$ are branched cobordant. A proof of this by different methods was known when $M_{1}, M_{2}$ and $N$ are orientable and $3 \leqslant d$ [Edmonds, 1979].

Definition. Let $\phi: M_{1} \rightarrow N$ and $\psi: M_{2} \rightarrow N$ be branched coverings of closed surfaces. Then $\phi$ and $\psi$ are branched cobordant if there is a 3-manifold $Q$ and a branched covering $\xi: Q \rightarrow N \times I$ such that $\partial Q=M_{1} \amalg M_{2}$ and $\xi \mid M_{1}=\phi$ and $\xi \mid M_{2}=\psi$. 
Let $H_{1}=D^{2} \times D^{1}$ denote the 3-dimensional 1-handle with attaching region $D^{2} \times \partial D^{1}$, where $D^{1}=[-1,1]$. The involution $\tau: H_{1} \rightarrow H_{1}$ given by $(x, y, z) \rightarrow$ $(x,-y,-z)$ interchanges components of the attaching region and defines a branched covering $H_{1} \rightarrow H_{1} / \tau$.

Let $M$ be a closed, connected surface. Then one obtains a cobordism $W$ from $M \times[0,1]$ by attaching a 1 -handle to $M \times\{1\}$. If $M$ is orientable, then $W$ may be orientable or nonorientable. The following is from [Berstein and Edmonds, 1979].

4.1. LemMA. Let $\phi: M \rightarrow N$ be a $d$-fold branched covering of closed, connected surfaces where $2 \leqslant d$. Let $W$ be as above and suppose $W$ is orientable if $N$ is orientable. Then $\phi$ extends to a branched cobordism $\Phi: W \rightarrow N \times[0,1]$.

Notice that $\Phi \mid \partial_{1} W$ has two more branch points than $\Phi \mid \partial_{0} W=\phi$.

Definition. A $d$-fold, simple, primitive branched covering $\xi: F \rightarrow G$ of closed surfaces is stable if $2 d-2 \leqslant d \chi(G)-\chi(F)$.

4.2. Lemma. Let $\xi_{1}, \xi_{2}: F \rightarrow G$ be $d$-fold, simple, primitive branched coverings of closed surfaces. If $\xi_{1}$ and $\xi_{2}$ are stable, then there exist homeomorphisms $f: F \rightarrow F$ and $g: G \rightarrow G$ such that $\xi_{2} \circ f=g \circ \xi_{1}$ and $g$ is isotopic to the identity.

Proof. From [Berstein and Edmonds, 1979] and the theorems of the previous section, one has homeomorphisms $f^{\prime}: F \rightarrow F$ and $g^{\prime}: G \rightarrow G$ such that $\xi_{2} \circ f^{\prime}=$ $g^{\prime} \circ \xi_{1}$. If $G$ is orientable, then $g$ may be chosen to be orientation preserving. Now it suffices to find homeomorphisms $\tilde{k}: F \rightarrow F$ and $k: G \rightarrow G$ such that $\xi_{2} \circ \tilde{k}=k \circ \xi_{2}$ and $k^{-1}$ is isotopic to $g^{\prime}$. For then taking $f=\tilde{k} \circ f^{\prime}$ and $g=k \circ g^{\prime}$ finishes the proof.

Suppose $\xi_{2}$ is a $d$-fold covering. Let $\xi: E \rightarrow S^{2}$ be a $d$-fold simple branched covering such that $\left|B_{\xi}\right|=\left|B_{\xi_{2}}\right|$. Let $p: G \times\{1,2, \ldots, d\} \rightarrow G$ be projection. Then $F^{\prime}=E \# G \# \cdots \# G$ is homeomorphic to $F$ and $G^{\prime}=S^{2} \# G$ is homeomorphic to $G$ and $\xi \# p: F^{\prime} \rightarrow G^{\prime}$ is equivalent to $\xi_{2}$. By construction of $\xi \# p$ it is clear that any homeomorphism of $G^{\prime}$ fixed on $S^{2}$ lifts to $F^{\prime}$. Now choose $k$ and $\tilde{k}$ appropriately.

4.3. ThEOREM. If $\phi: M_{1} \rightarrow N$ and $\psi: M_{2} \rightarrow N$ are $d$-fold, simple, primitive branched coverings between closed, connected surfaces, then $\phi$ and $\psi$ are branched cobordant.

Proof. By the first lemma $\phi$ and $\psi$ extend to branched cobordisms $\xi_{1}: W_{1} \rightarrow N \times$ $\left[0, \frac{1}{2}\right]$ and $\xi_{2}: W_{2} \rightarrow N \times\left[\frac{1}{2}, 1\right]$ such that $\xi_{1} \mid \partial_{1 / 2} W_{1}$ and $\xi_{2} \mid \partial_{1 / 2} W_{2}$ are equivalent, stable, primitive simple branched coverings. By Lemma 4.2 there exist homeomorphisms $f$ and $g$ yielding $\xi_{1} \cup \xi_{2}: W_{1} \cup_{f} W_{2} \rightarrow N \times\left[0, \frac{1}{2}\right] \cup_{g} N \times\left[\frac{1}{2}, 1\right]$. Since $g$ is isotopic to the identity, $N \times\left[0, \frac{1}{2}\right] \cup_{g} N \times\left[\frac{1}{2}, 1\right]$ may be fibered as desired.

Theorem 4.3 is true even if $\phi$ and $\psi$ are not necessarily simple and primitive. This will be proved next.

Definition. Branched coverings $\phi, \psi: M \rightarrow N$ are b-homotopic if there is a level preserving homotopy $H: M \times I \rightarrow N \times I$ from $\phi$ to $\psi$ such that $H_{t}$ is a branched covering for all $t$.

Note that $H$ is a branched covering. In [Berstein and Edmonds, 1979] it is shown that any branched covering of compact surfaces is $b$-homotopic to a simple branched covering. Hence any branched covering of closed surfaces is branched cobordant to a simple branched covering. 


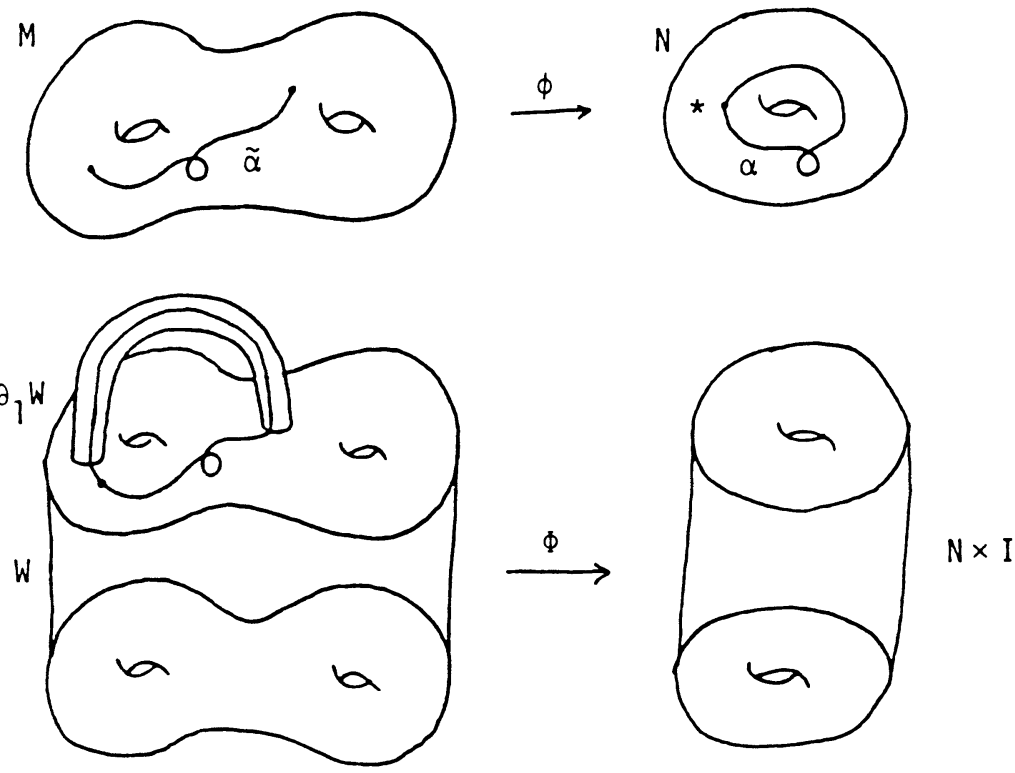

FIGURE 4.1

4.4. LEMmA. Let $\phi: M \rightarrow N$ be a branched covering of closed, connected surfaces. Then $\phi$ is branched cobordant to a primitive, simple branched coverings.

Proof. Let $\alpha$ be any loop in $N$ representing an element of $\pi_{1}(N) \backslash \operatorname{Image}\left(\phi_{*}\right)$. Then it suffices to show how to extend $\phi$ to a branched cobordism $\Phi: W \rightarrow N \times[0,1]$ such that $[\alpha] \in \operatorname{Image}\left(\left(\Phi_{*} \mid \partial_{1} W\right)_{*}\right)$. A judicious placement of the 1-handle in $W$ will allow $\alpha$ to lift to a loop in $\partial_{1} W$ (see Figure 4.1).

4.5. THEOREM. If $\phi: M_{1} \rightarrow N$ and $\psi: M_{2} \rightarrow N$ are $d$-fold branched coverings between closed, connected surfaces, then $\phi$ and $\psi$ are branched cobordant.

Notice that in the above theorem if $\phi$ and $\psi$ are simple, then the cobordism may be chosen to be simple. If $M_{1}, M_{2}$ and $N$ are oriented and $\phi$ and $\psi$ are orientation preserving, then $W$ is orientable and may be chosen with orientation such that $\partial W=M_{1} \amalg-M_{2}$, where $-M_{2}$ denotes $M_{2}$ with opposite orientation.

\section{REFERENCES}

I. Berstein and A. Edmonds, On the construction of branched coverings of low-dimensional manifolds, Trans. Amer. Math. Soc. 247 (1979), 87-124. , On the classification of generic branched coverings of surfaces, Illinois J. Math. 28 (1984), 64-82.

A. Clebsch, Zur Theorie der Riemann'shen Flächen, Math. Ann. 6 (1873), 216-230.

A. Edmonds, Deformation of maps to branched coverings in dimension three, Math. Ann. 245 (1979), 273-279.

A. Hurwitz, Über Riemann'sche Flächen mit gegebenen verzweigungspunkten, Math. Ann. 39 (1891), $1-60$.

J. Lüroth, Note über Verzweigungsschnitte und Querschnitte in einer Riemann'schen Fläche, Math. Ann. 4 (1871), 181-184.

R. Skora, Maps between surfaces, Ph.D. Dissertation, University of Texas at Austin, 1984.

DePaRTMENT OF MATHEMATICS, INDIANA UNIVERSITy, BLOOMINGTON, INDIANA 47405 